\title{
Culture-broker and medical decoder: contributions of caregivers in American Indian cancer trajectories
}

Felicia Schanche Hodge, DrPH, ${ }^{\text {ab }}$ Mary Cadogan, DrPH, RN, GNP-BC, ${ }^{a}$ Tracy Line Itty, MPH, ${ }^{a}$ Angela Williams, FNP-C, MSN, ${ }^{a}$ and Arneta Finney, FNP-C, MSN, PHN ${ }^{a}$

${ }^{\mathrm{a}}$ School of Nursing and Public Health ${ }^{\mathrm{b}}$ Fielding School of Public Health, University of California, Los Angeles

Background Caregivers play a special role in the management and control of cancer-related pain. For American Indians with cancer, caregivers can contribute to patient education, medication compliance, and can facilitate communication between the patient and the provider and the patient and the family.

Objective To identify the role(s) of caregivers of American Indian cancer survivors.

Methods As a part of a large randomized intervention designed to improve barriers to cancer symptom management, 13 focus groups were held among American Indian cancer survivors and their caregivers at Southwest reservations and urban sites. Focus groups, audiotaped and transcribed, used constant comparative methods in the analysis of caregiver dialogues.

Results Caregivers are patient educators and provider culture-brokers and their communication strategies use a combination of cultural and conventional strategies in their care of American Indian cancer patients. Cultural communication styles include "talk stories" (storytelling), group (talking circles), and dialogue to manage cancer pain, educate the patient and community, and to protect the patient from stigma, reduce barriers to care, and provide support to patients and families. Active discussion with providers "re-packaged" the patient's reporting/responses to specific clinical measures (pain measure scores) and identified the need for pain medication and compliance-related issues.

Limitations Findings are not generalizable to the American Indian population outside of the sites and focus groups from which data were collected.

Conclusions Caregivers are "cultural brokers" who inform providers of the cultural nuances associated with American Indian patient care. However, caregivers voiced that cultural restriction for not discussing illness openly was a sanction and an important barrier.

Funding National Cancer Institute, NIH, grant RO 1 CA 115358

$\mathrm{C}$ aregivers have received increasing attention in the literature as their role in providing support and care to severely ill or disabled individuals has become recognized as essential to the palliative care of those undergoing medical treatment and long-term care. ${ }^{1}$ Caregivers can fill many roles, such as transporter, companion, housekeeper, cook, and nurse. ${ }^{2}$ They can also serve as liaisons between the patient and health care providers in the management and control of cancer-related pain. $^{3}$

The National Family Caregivers Association reports that more than 50 million caregivers provide long-term care for loved ones with disabilities or chronic illnesses during a given year. ${ }^{1}$ According to Sawchuk, ${ }^{3}$ the multifaceted duties of caregivers generally involve medical, physical, functional, emotional, and financial support. However, Gillick ${ }^{4}$ reports the role of caregivers has expanded beyond assisting with activities of daily living (ie, feeding, bathing, and transfers), preparation of special diets, and administration of oral medications. Caregivers are more often trained to provide specialized care to patients, such as wound care, administration of intravenous fluids and parenteral medications, management of feeding tubes, tracheostomy tubes, and medical equipment such as mechanical ventilators and dialysis apparatuses. The increase in the level of care provided by caregivers can have an enormous economic impact, costing as much as $\$ 450$ billion in $2009,{ }^{1}$ and a reported value of an estimated $\$ 350$ billion in 2006 in unpaid caregiver services. ${ }^{5}$ One report estimates that the average caregiver can provide up to 20-25 hours of unpaid care per week. ${ }^{6}$

For minority populations, caregivers are especially critical in aiding the cancer patient through the health care system. For American Indians with a cancer diagnosis, caregivers do more than aid in

Accepted for publication February 22, 2016. Correspondence: Felicia Schanche Hodge, DrPH; fhodge@sonnet.ucla.edu. Disclosures: The authors report no disclosures or conflicts of interest. JCSO 2016;14:221-228. (2) 2016 Frontline Medical Communications. doi: 10.12788/icso.0254. 
activities of daily living (ADL) and symptom management - they can assist in overcoming language barriers, educating the family and community, and promoting culturally sensitive care. In American Indian communities, support networks of family and friends who understand the unique living situation of American Indians living in rural areas can provide culturally competent longterm care. ${ }^{1}$ This paper explores the roles of caregivers of American Indian cancer patients as they facilitate the long-term care of the patient, as well as the interaction between the patient and the provider. The need for such a study as reported in this manuscript is particularly strong to inform health care providers on the supportive role of the caregiver, and to provide information on the types of information and care provided by caregivers - that is greatly needed by both American Indian cancer patients and their communities.

\section{Methods}

A National Cancer Institute-supported randomized clinical trial to improve barriers to cancer symptom management among American Indians was held in the state of Arizona in 2006-2012. The study included individual cancer patient interviews $(n=20)$, focus groups $(n=88$ in 13 focus groups), and a pre- and post-test intervention trial of 200 recruited subjects. The interviews and focus groups informed the development of an educational toolkit ${ }^{7}$ that was designed to improve cancer symptom management by reducing barriers, increasing knowledge, and improving pain relief.

The focus groups consisted of a convenience sample of 88 caregivers of Southwest American Indian cancer patients who participated in 13 focus group discussions on cancer symptom management. Each focus group consisted of 4-15 mutually exclusive participants; focus group members participated in only 1 of the 13 sessions. Inclusion criteria for caregivers were: age 18 years or older; caregiver (including family and friends) of an American Indian cancer patient; and English speaking. Focus groups were held at 2 reservation sites and 2 urban clinics in the state of Arizona. Tribal clinic meeting rooms or offices were provided by the tribes and urban clinic staff for use by the research team. Participants were recruited through informational flyers that were posted throughout the clinic areas. The flyers indicated the purpose of the study, the site for the focus groups, and the eligibility criteria. Interested eligible individuals were instructed to call a number to enroll in the study. The sessions were held in the spring and fall months of 2010, were 1.5-2 hours long, included patients and caregivers, and were audiotaped and transcribed verbatim. Incentives, in the form of $\$ 35$ gift cards, were offered to all participants to cover travel and expenses. Information was gathered on participants' experience in their role as caregivers to American Indian cancer patients.
The project focus group facilitator obtained consent from each participant prior to enrollment. The study received written approval from each tribe/urban group as well as from the University of California, Los Angeles (UCLA) and the Phoenix Area Indian Health Service institutional review boards, which oversee the protection of human participants for the service area tribes/organizations. Participants were provided a copy of the consent form and were told that their participation was voluntary, that health care services were not contingent upon their participation, and that they did not have to answer any question(s) that they did not want to or that they found uncomfortable. Further, focus group participants were told that their responses were confidential, as their name and other identifiers would not be recorded. Information was shared about the goals of the study, their role in and length of their participation, as well as the background, work status, and research interest/background, and contact information of the research team.

The research team consisted of highly trained faculty and staff at UCLA as well as trained American Indian facilitators from each of the study sites (1-2 facilitators per site). The focus groups were held by the senior researcher member assisted and coordinated by facilitators. These facilitators completed training specific to their roles in the focus groups. A detailed focus group protocol was developed and used by the facilitators and researchers, consisting of information on their roles, focus group logistics, guiding questions, probing questions, and how to facilitate problems or issues that may arise. The role of the facilitator was to coordinate the logistics, advertise the site of the focus group, assist in the enrollment and consenting process, audiorecording the sessions, and assisting the researcher during the course of the focus group session. Guiding and probing focus group questions are shown in Table 1.

\section{Analysis}

Transcribed focus group sessions were analyzed using grounded theory methodology $y^{8}$ to identify major codes and categories in the data. Transcripts were read in their entirety by the research team and then excerpt-by-excerpt coding was conducted by 2 researchers to capture the meaning expressed in each excerpt. A qualitative data analysis software (ATLAS.ti) was used to categorize the caregiver ${ }^{4}$ data and to manage the developing themes. Codes were grouped by importance, related concepts, and frequency as major themes emerged from the data. Following an initial identification of themes, a second investigator independently reviewed the categorized codes and key themes. Code description and categorization were discussed and modified until agreement among the entire research team was achieved (Table 2).

The results of the data analysis, using grounded theory methodology, formed a theoretical construct of re-packag- 
TABLE 1 Guiding and probing focus group questions

\section{Focus group 1: Experiences with cancer}

Today we are going to talk about your patient's health, your experiences, and explanations for symptoms with regard to illness. [Questions in caps are probes.]

1. Tell me about the diagnosis. WHEN WERE THEY DIAGNOSED? HOW DID THEY/YOU GET TO THE DOCTOR? WHAT HAPPENED?

2. What do you think caused it? WHY DID THAT CAUSE IT?

3. When did it start? WHY DID IT START? HOW DID IT START? WHAT DO YOU THINK CAUSED IT? DO YOU TELL PEOPLE ABOUT THE ILLNESS/ WHAT DO YOU CALL IT?

4. What does your sickness do to the patient? BODY, THEIR LIFE? WHAT DOES IT DO TO FAMILY? HOW DOES IT AFFECT DAILY LIVING?

5. What do you think will happen with the sickness? WHAT DO YOU THINK WILL/WANT TO HAPPEN?

6. What do you worry/fear most about the illness? WHAT ARE THE CHIEF PROBLEMS THAT YOUR SICKNESS HAS CAUSED? HOW ABOUT PAIN? HOW ABOUT FATIGUE? HOW ABOUT LOSS OF FUNCTION? HOW ABOUT DEPRESSION? DO YOU HAVE THE "BLUES", OR OTHER PROBLEMS?

7. How is the sickness treated? HOW DO YOU THINK IT SHOULD BE TREATED?

8. What other things are done to treat it? WHAT KINDS OF ASSISTANCE IS NEEDED?

9. What are the most important results you hope to get from the treatment?

10. Are there any taboos/ stigmas associated with the illness? CAN YOU DESCRIBE THEM?

\section{Focus group 2: Symptom measurement and symptom management}

Today we are going to talk about cancer symptoms, such as pain, fatigue, loss of function, and depression, and we will discuss how these symptoms are measured and managed. [Questions in caps are probes.]

Pain The first symptom we will talk about is pain.

1. There may be many ways to measure what we feel. Take a look at this pain scale - have you seen it before? WHEN IT WAS USED?

2. Are you able to use the scale? WHY NOT?

3. Does it help to explain pain? HOW?

4. If you or the patient were to come up with another way to explain pain -how would you do it?

5. Did you talk to the doctor or nurse about the pain? WHAT HAPPENDED?

Fatigue The next symptom we are going to talk about is fatigue.

1. Did you notice that patient felt tired? CAN YOU DESCRIBE IT?

2. What did you do to help with this feeling of tiredness?

3. Did you talk to the doctor or nurse about it? WHAT HAPPENED?

4. Take a look at this scale - let me know if any questions are hard to answer-or if you do not like any of them. WHY - WHAT CHANGES WOULD YOU SUGGEST? DO THESE QUESTIONS HELP TO EXPLAIN OR MEASURE FATIGUE? WHY/ WHY NOT? 
Continued from previous page

Functionality The next symptom we are going to talk about is loss of function.

1. Does patient have any loss of functioning due to your cancer diagnosis? PLEASE DESCRIBE.

2. What did you do to help with this loss of function?

3. Did you talk to the doctor or nurse about it? WHAT HAPPENED?

4. Take a look at this scale - let me know if any questions are hard to answer - or if you do not like any of them. WHY - WHAT CHANGES WOULD YOU SUGGEST? DO THESE QUESTIONS HELP TO EXPLAIN OR MEASURE THE LOSS OF FUNCTION? WHY?

Depression The next symptom we are going to talk about is depression.

1. Did you notice that the patient may have felt sad or had the 'blues'? HOW OFTEN?

2. Can you describe the depression? WHEN WOULD IT HAPPEN? WHAT DID YOU DO ABOUT IT?

3. Did you talk to the doctor or nurse about it? WHAT HAPPENED?

4. What medicines or treatment is taken for depression?

5. Take a look at this scale - let me know if any questions are hard to answer - or if you do not like any of them. WHY - WHAT CHANGES WOULD YOU SUGGEST? DO THESE QUESTIONS HELP TO EXPLAIN OR MEASURE LOSS OF FUNCTION? WHY?

6. If you were to come up with another way to explain depression - how would you do it? WHAT WORDS WOULD YOU USE?

ing of the cancer experience. The caregiver was a key player in efforts to improve patient and provider understanding of the cultural constructs of the cancer experience, barriers to care, and the need for medication or issues surrounding treatment compliance or understanding.

\section{Results}

The majority of caregivers (70\%) was comprised of family members who self-identified as being a member of a Southwest United States tribe. Sixty percent of them were women and 40\% were men. All of the caregivers reported that they spoke English, and all but 1 spoke another language (eg, Spanish, Navajo, Apache, Hopi, Tohono O'odham, or Yaqui).

Caregivers used a combination of cultural and conventional strategies in their care of American Indian cancer patients. An understanding of culture, defined as a set of shared beliefs and values that shape customs, traditions, behaviors and beliefs, ${ }^{9}$ was illustrated within the context of the cancer experience by the caregivers during the focus groups. Cultural definition of illness, perceptions of symptoms, treatment compliance, health-seeking behaviors, as well as perceived health and prevention service needs, were established. Caregivers discussed their role as a medical "decoder" for their patients and family members, in addition to acting as a "culture broker" to primary care doctors, oncologists, and nurses.

\section{Caregiver as medical decoder}

Communicating with the patient often required "decoding" or making sense of the medical terms and describing cancer in both etiology and trajectory terms of the disease. Focus group participants reported that caregivers become well versed in basic medical terminology - specifically oncology - out of necessity, because patients depended on them to interpret medical findings, as well as to support and encourage the treatment protocol. The caregiver typically spoke the native language of the patient, or would find someone to interpret for them. Several caregivers commented about translating during medical visits. Items translated included information of impending death, information on treatment such as the schedule for chemotherapy, or the name of the medication prescribed by the physician, and the treatment regimen. One caregiver shared a situation where the patient wanted to have provider's information explained to him,

$\ldots$ in the end when he was told [by the provider] that he had terminal cancer, he asked people to explain it to him, so I told him in Apache what the doctors said and what it meant and the things they told him. Later on he just lowered his head and said 'Adalezy, there is no hope'.

Sharing the diagnosis with the family and translating the meaning of cancer, expected trajectory of the disease, and 
TABLE 2 Description of coding, identification of major and minor focus group themes

Themes

Coding category
Major

Fatalism - meant to be

We don't talk about it with nuclear and extended family members

\section{Minor}

Cannot alter your life course. How to live with cancer diagnosis.

Caregiver as interrupter. Cannot burden others. Privacy.

Communication

Respect for health care providers

Correcting or asking questions of providers is shameful - disrespectful. Caregiver to mediate cultural divide.

\section{Symptom \\ management}

Barriers
Manage alone - in silence or with caregiver

Financial, transportation, translation
Do not complain. Caregiver is patient's voice. Caregiver can help patient to understand - decoding.

Not enough money for household, travel to hospital. Cultural barrier strong. preparation for death and dying are major roles of the caregiver. A caregiver explained,

\begin{abstract}
My doctor told me ... that I have to share with family members, like cousins or anyone who was close ... notifying the family that she might die that week, and that the family be prepared, and to know that she will be gone.
\end{abstract}

Caregivers are also knowledgeable of the specific needs of gender or age groups. A caregiver commented,

I think they need time to grieve ... men hide problems, my father was very sick, he did not tell us what is wrong till the end. In addition, caregivers facilitated introduction and discussion of services often unfamiliar to members of the community. There is another thing ... hospice ... there is a lot of people [that have cancer] that don't know about that ... and they deal a lot with those patients. A lot of people are not informed of the things that are available to them ... the different types of services.

\section{Caregiver as constant companion and helper}

As caregiver family members (or individuals close to the family) took on the responsibility of staying with the patient at all times, they assumed the role of the mother, interpreter, driver, cook, housekeeper, and health care aide. Most participants reported living with the cancer patient and would provide transportation to medical appointments particularly "if they don't know anyone going [they will] volunteer to go with them." A caregiver shared,

... sometimes family have to stay overnight, sometimes you have the van take you to IHS [Indian Health Service clinic or hospital], but they don't stay around to take you back, so you have to stay overnight and make arrangements.

The cancer diagnosis and treatment took quite a toll on family roles and dynamics, changing relationships and expectations,

\begin{abstract}
For my aunt, I know it disrupted her life because she was unable to do anything for herself, she couldn't be a mother ... I know it caused a lot of pressure on the family, short tempers, and it also brought the family a little closer together. At the time I wasn't really actively involved with that side of the family, but then after the sickness, it brought us together and we were always around her - being around her as much as possible, rotating, helping her with household chores...
\end{abstract}

This multipurpose role of the caregiver was described as a rewarding, yet a distressing job. With little or no training, the caregiver looked after the daily needs of the patient, while observing a decline in health. One caregiver said, "I am having a hard time with this ... helping people, working with people fighting for their life." Another said, "I have gotten to know them very well. Watch them struggle with their condition." Other caregivers mirrored these sentiments,

Then that year, [I] come to know [the patient's] experience and to see her day in and day out, knowing her good days and bad days. ... I know she does not want any preferential treatment ... she wants to be treated normal, and yet sometimes I see her face and I know she is sick, and so it is really hard. How do you deal with that? So I find myself in a real situation.

It is the family [caregivers] that takes care of them day in and day out, so they see a lot of things going on there. With my uncle... [he was] up at all hours of the night and [l saw] how uncomfortable he was and the pain he was in. Those are the kinds of things ... witness ... however in 
my position you see the individuals. I have gotten to know them very well. Watch them struggle with their condition.

\section{Caregiver as cultural broker}

The caregivers who reported that they were most successful in their roles acted as "cultural brokers" - they were knowledgeable of the culture-bound illness beliefs, fears, and traditional treatments and ceremonies. Understanding the cultural constructs of illness, having a strong understanding of the illness beliefs, particularly the onset and trajectory of cancer and cancer pain management are important attributes of the caregiver.

One of the most important cultural barriers that caregivers addressed in our study was that American Indian cancer patients tend to not bring their burdens home to the family or community - isolating them to suffer in silence.

\begin{abstract}
... when you start seeing changes in them, starting to get sick, not being able to do things that they did. Especially here [on the reservation], they are really strong people, they are not used to having anybody help them. Like my uncle, they are real strong Apache man and real private people. Not used to seeing people care for them [such a nurses and doctors or other family members]. Part of that respect, they keep to themselves [so they do not share many things with the providers].
\end{abstract}

The cultural value of self-reliance and the importance of respect, privacy, and personal space (comfort with being touched or handled by strangers) among tribal groups are important and the caregiver must abide by those values and to translate the cultural values to the medical care personnel. One caregiver shared, "cancer and diabetes are some of the things that they don't talk about ... it is too personal to talk about it." A common phrase among the caregivers was "we don't talk about it" to spare additional hardship on family members because "we don't bring our burdens home." These beliefs and communication restrictions were shared with the medical providers so they could understand why patients do not talk about their illness and why they do not often share it with their family members,

She has been sick all this time but she won't even let us know ... even from the family ... she was hiding it. I went to her house, she was sick, I told her to go to the clinic. She was scared to go down to the hospital, they were going to send her to Phoenix. But she didn't want to go ... so one of the nurses told me if I could go with her, I said okay, so I took off with her. But she did not want to go by herself.

... my dad was like that too, he was kind of traditional. I know a lot of the older people are really traditional ... My dad, he passed on already so you don't talk about it
... They said once they are gone, they are gone ... they don't talk about them.

I know my uncle admitted he knew there was something wrong. He was smoking. There were certain things that were happening. I think they know, but they don't talk about it. I think they know but keep to themselves.

The cultural restriction for not discussing illness openly was an important barrier to patient communication. Overcoming this delicate situation became an important role for the caregivers for the management of cancer pain and other symptoms. Caregivers tell of running "into people I am talking with and they ask me if I know anything about certain diseases, we talk about what we both know." Talking about cancer was taboo thus fearful as saying the word "cancer" may bring forth the disease, because the fear ... of the word itself was something the community would not acknowledge."

Another topic often shared with providers was the barrier to cancer care faced by American Indian patients. Caregivers identified such barriers as lack of transportation, lack of knowledge regarding resource availability, and the expenses associated with hospitalization and death and dying. One caregiver explained,

... most of the household money goes to her travel ... and we have issues at home ... pay for bills ... propane, electricity ... some of us have food stamps so we have to pitch in to get food and stuff ... because all that money goes to ... she gets ... goes to her transportation [to the hospital]. We don't have money for household stuff. A lot of us don't work because there are no jobs around here.

When she was in the hospital, I know it was a financial burden because she was in Phoenix and traveling from here to Phoenix was expensive, especially on gas. And not having family in the Phoenix area was another problem because we had to pull money together to get a room, gas money, and food, but that was toward the end when she was about to go.

Using a combination of cultural and conventional strategies in their care of American Indian cancer patients allows caregivers to disseminate information on cancer symptom management, all the while being careful in discussing illness diagnosis and treatment when the opportunity arises. One such opportunity was through the direction of the patient's physician to notify family and others of the pending passing of the patient and "that I have to share with family members." At another time, the nurse directed the caregiver to transport the patient to the hospital, thus giving permission to relay to others the need for medical care for the patient. Packaging the management of cancer pain within a cultural 
context educates the patient and community, and has the added benefit of reducing barriers to care and providing support to patients and families. Repackaging the cultural constructs of the cancer patient's illness beliefs and culturebound environment for the nurses and physicians educates the providers as to the barriers, illness beliefs, and taboos or restrictions within the community or family unit that can explain or facilitate the barriers or trajectory of steps involved in the illness experience.

\section{Discussion}

Communication between patients and their health care providers and caregivers is an important part of the cancer experience. This paper reports on qualitative findings of a study looking at the pain experience of American Indian cancer patients and survivors, particularly in the areas of communication with family and health care providers. Analysis based on grounded theory techniques was used to assess the communication patterns of American Indian cancer patients/survivors residing in the Southwest. Twenty individual interviews and 13 focus groups examined key themes in cancer pain communication. The results were categorized into communication with nuclear/extended family members and communication with health care providers. Within these 2 categories, 3 themes emerged: "we don't talk about it" (expectations not to burden others); respect for health care providers (correcting or asking questions of providers is shameful); and the cancer experience is "meant to be" (fate cannot be altered). Cultural factors, such as perceptions of fatalism and respect for others, greatly influence the communication patterns of American Indian patients/ survivors, resulting in the minimizing of reports and complaints regarding cancer pain. Understanding communication patterns and the reasons for reduced communication reported by cancer patients/survivors is important for health care providers, caregivers, and others providing treatment and control of cancer pain.

With a foot in both worlds, the role of the caregiver is expansive and critical to understanding the specific culture of the cancer patient and to be familiar with the world of oncology. Caregivers for American Indian patients grapple with many issues, particularly those related to cultural constructs ${ }^{10}$ that can lead to inadequate management of cancer pain. This paper reported on the strategies employed by caregivers of American Indian cancer patient as they maneuver the cancer trajectory with their patients. Caregivers taking part in this study reported acting as medical decoders to interpret the medical jargon and provider instructions/ diagnosis for American Indian patients so that they can manage their cancer symptoms and receive needed medical care - and also as intermediary culture-brokers between the patient and the health care professionals.

The literature demonstrates that culture-bound beliefs can act as barriers to cancer care. ${ }^{11}$ Caregivers report that many American Indians do not share their illness with others because they are private individuals who are uncomfortable and unable to "bring their burdens home." Many limit communication with medical providers because of discomfort and/or the preference for privacy. With more than 500 American Indian languages and as many tribes in the United States, effective patient-provider interaction is critical to good patient care. Caregivers can assist in overcoming language barriers, contribute to pain management, educate the family and community, and can facilitate communication between the patient and the provider.

Culture plays an important role in the extent to which recommended treatment is received and accepted by American Indians. The communication and interaction by the provider must take cultural constructs into account, as it can have significant influence on health behaviors. Thus understanding the culture and the influence it has on symptom perceptions and the cause of illness has implications for improving patient-provider communications and improving caregiver management of cancer symptoms among American Indian communities, as well as other marginalized communities who share similar cultural barriers.

Limitations of our study include issues of geography, translation, and study size. Because we restricted our study to American Indians in the Southwest United States, we cannot generalize findings beyond that region. In addition, we found that most participants spoke their tribal language or Spanish in addition to English and we could not validate some of the discussions as interpreters informed us that they could not translate some of the terms into English. And lastly, the study population consisted of American Indian cancer patients and their caregivers - those who declined participation in the study (6 adults) did not give feedback as to why they declined to participate. Our study size was relatively small, given the population size of American Indians in the Southwest.

\section{Acknowledgment}

The contents of this manuscript are solely the responsibility of the authors and do not necessarily represent the official views of the NIH/ NCI or the Indian Health Service. 


\section{Original Report}

\section{References}

1. Spencer SM, Goins RT, Henderson J, Wen Y, Goldberg J. Influence of caregiving on health-related quality of life among American Indians. J Am Geriatr Soc. 2013;61:1615-1620.

2. Yen P, Benson W. Caregiving in Indian country: Conversations with family caregivers. National Association of Chronic Disease Directors. http://www.chronicdisease.org/?page=Briefs . Published 2010. Accessed April 24, 2016.

3. Sawchuk CN, Van Dyke E, Omidpanah A, Russo JE, Tsosie U, Buchwald D. Caregiving among American Indians and Alaska Natives with cancer. Supp Care Cancer. 2015;23:1607-1614.

4. Gillick MR. The critical role of caregivers in achieving patient-centered care. JAMA. 2013;310:575-576.

5. AARP. Family caregiving valued at $\$ 350$ billionr. htty://www.aarp. org/about-aarp/press-center/info-2007/family_caregiving.html. Released June 25, 2007. Accessed April 22, 2016.

6. Goins RT, Spencer SM, McGuire LC, Goldberg J, Wen Y, Henderson JA. Adult caregiving among American Indians: the role of cultural factors. Gerontologist. 2011;51:310- 320.
7. Hodge FS, Line TL, Cadogan ME, Martinez F. 'Weaving balance into life': Development and cultural adaptation of a cancer symptom management toolkit for Southwest American Indians. J Cancer Surviv. 2012;6:182-188.

8. Glaser B, Strauss A. The discovery of grounded theory: strategies for qualitative research. Chicago, IL: Aldine.

9. Haddad L, Smith CM. Relevance of culture and values for community/public health nursing. In Maurer FA, Smith CM, eds.

Community/public health nursing practice: Health for families and populations. 5th ed. St Louis, MO: Elsevier; 2013:266-297.

10. Jimenez N, Garroutte E, Kundu A, Morales L, Buchwald D. A review of the experience, epidemiology, and management of pain among American Indian, Alaska Native, and Aboriginal Canadian peoples. J Pain. 2011;12:511-522.

11. Itty TL, Hodge F, Martinez F. Shared and unshared barriers to cancer symptom management among urban and rural American Indians. J Rural Health. 2014;30:206-213. 